\title{
Identification of the Poisson and Martin boundaries of orthogonal discrete quantum groups
}

\author{
By StefaAn VAes ${ }^{(a, b)}$ And Nikolas Vander $\operatorname{Vennet}^{(\mathrm{b})}$ \\ Journal of the Institute of Mathematics of Jussieu 7 (2008), 391-412.
}

(a) CNRS; Institut de Mathématiques de Jussieu; Algèbres d'Opérateurs; 175, rue du Chevaleret; F-75013 Paris (France)

(b) K.U.Leuven; Department of Mathematics; Celestijnenlaan 200B; B-3001 Leuven (Belgium)

e-mail: stefaan.vaes@wis.kuleuven.be,nikolas.vandervennet@wis.kuleuven.be

\begin{abstract}
The Poisson and Martin boundaries for invariant random walks on the dual of the orthogonal quantum groups $A_{o}(F)$, are identified with higher dimensional Podleś spheres that we describe in terms of generators and relations. This provides the first such identification for random walks on non-amenable discrete quantum groups.
\end{abstract}

\section{Introduction}

Group invariant random walks on countable groups have been studied intensively and the identification of the associated Poisson and Martin boundaries is a natural problem. We refer to [12] for an excellent survey. The study of random walks on discrete quantum groups was initiated by Biane [3] who considered duals of compact groups and obtained a theory parallel to the theory of random walks on discrete abelian groups.

Random walks on arbitrary discrete quantum groups (i.e. duals of Woronowicz' compact quantum groups [19]) and their Poisson boundaries were introduced by Izumi in [10], motivated by the study of infinite product actions of compact quantum groups. In [10], Izumi identified the Poisson boundary of the dual of $\mathrm{SU}_{q}(2)$ (see [20]) with the Podleś sphere [15]. Later, Neshveyev and Tuset [14] associated a Martin boundary with a random walk on a discrete quantum group and proved that the Martin boundary of the dual of $\mathrm{SU}_{q}(2)$ is still given by the Podleś sphere. This generalized Biane's work [5] on the dual of SU(2).

The Poisson boundary for the dual of $\mathrm{SU}_{q}(n)$ was computed by Izumi, Neshveyev and Tuset in [11], but its Martin boundary remains mysterious. Partial results on the Martin boundary for the dual of SU(n) were obtained by Biane [4] and Collins [7].

The discrete quantum groups appearing in the previous paragraphs are all duals of classical groups or their $q$-deformations. A quite different class of compact quantum groups was introduced by Van Daele and Wang $[18]$ and studied by Banica $[1,2]$. In this paper, we identify the Poisson and Martin boundary for the dual of the orthogonal compact quantum groups $A_{o}(F)$.

The orthogonal compact quantum groups $A_{o}(F)$ are quite peculiar. Given an $n$ by $n$ matrix $F$ satisfying $F \bar{F}= \pm 1$, they are defined as the compact quantum group generated by an $n$-dimensional unitary representation $U$ satisfying $U=F \bar{U} F^{-1}$. On the one hand, their representation theory is similar to the one of $\mathrm{SU}(2)$, both having the same fusion rules. More precisely, every $A_{o}(F)$ is monoidally equivalent to an $\mathrm{SU}_{q}(2)$ for a uniquely determined $q$, in the sense of [6]. The quantum groups $\mathrm{SU}_{q}(2)$ appear as $A_{o}(F)$ for $F$ a 2 by 2 matrix. On the other hand, once $F$ is at least 3 by 3 , the dimensions of the irreducible representations of $A_{o}(F)$ start growing exponentially, yielding a very different operator algebraic behavior. In a sense, the operator algebras associated with $A_{o}(F)$ for $F$ at least 3 by 3 , share several properties with the free group $\mathrm{C}^{*}$ - and von Neumann algebras, see [16]. In particular, the dual discrete quantum group becomes non-amenable. As such, for the first time, Poisson and Martin boundaries of a non-amenable quantum group are identified.

In Theorem 5.2, the Poisson boundary for the dual of $A_{o}(F)$ is identified with a kind of 'higher dimensional Podleś sphere', that we describe in terms of generators and relations. In Theorem 6.1, it is shown that the Martin boundary for the dual of $A_{o}(F)$ can be identified with the $\mathrm{C}^{*}$-counterpart of these higher dimensional Podleś spheres. 
Our method to obtain the Poisson boundary for the dual of $A_{o}(F)$ goes as follows. We exploit the monoidal equivalence of $A_{o}(F)$ and $\mathrm{SU}_{q}(2)$ in order to reduce the identification problem to a purely $\mathrm{SU}_{q}(2)$-problem. The latter is solved invoking Izumi's computation for the Poisson boundary of the dual of $\mathrm{SU}_{q}(2)$ (see [10], or the alternative approach in [11]). The Martin boundary is obtained by using a result of [16], allowing to deduce the Martin boundary from the Poisson boundary. Altogether, our proofs depend on the known computation for the Poisson boundary of the dual of $\mathrm{SU}_{q}(2)$, but do provide an alternative method to identify the Martin boundary for the dual of $\mathrm{SU}_{q}(2)$ (as was done in [14]).

The method of this paper to identify the Poisson boundary for the dual of $A_{o}(F)$ by using the notion of monoidal equivalence suggests that there is a general way to describe the behavior of Poisson boundaries when passing to monoidally equivalent quantum groups. This will be the subject of a forthcoming paper of the second author.

\section{Preliminaries}

Consider a subset $S$ of a $\mathrm{C}^{*}$-algebra. We denote by $\langle S\rangle$ the linear span of $S$ and by $[S]$ the closed linear span of $S$. We use the notation $\omega_{\eta, \xi}(a)=\langle\eta, a \xi\rangle$ and we use inner products that are linear in the second variable.

We use the symbol $\otimes$ to denote several types of tensor products. In particular $\otimes$ denotes the minimal tensor product of $\mathrm{C}^{*}$-algebras, but it also denotes the tensor product of Hilbert spaces and von Neumann algebras. From the context, it will always be clear what we mean. We also make use of the leg numbering notation in multiple tensor products: if $a \in A \otimes A$, then $a_{12}, a_{13}, a_{23}$ denote the obvious elements in $A \otimes A \otimes A$, e.g. $a_{12}=a \otimes 1$.

\section{Compact quantum groups}

We give a brief overview of the theory of compact quantum groups which was developed by Woronowicz in [19]. We refer to [13] for a survey of basic results.

Definition 1.1. A compact quantum group $\mathbb{G}$ is a pair $(C(\mathbb{G}), \Delta)$, where

- $C(\mathbb{G})$ is a unital $\mathrm{C}^{*}$-algebra;

- $\Delta: C(\mathbb{G}) \rightarrow C(\mathbb{G}) \otimes C(\mathbb{G})$ is a unital *-homomorphism satisfying the co-associativity relation

$$
(\Delta \otimes \mathrm{id}) \Delta=(\mathrm{id} \otimes \Delta) \Delta
$$

- $\mathbb{G}$ satisfies the left and right cancellation property expressed by

$$
\Delta(C(\mathbb{G}))(1 \otimes C(\mathbb{G})) \text { and } \Delta(C(\mathbb{G}))(C(\mathbb{G}) \otimes 1) \text { are total in } C(\mathbb{G}) \otimes C(\mathbb{G}) .
$$

Remark 1.2. The notation $C(\mathbb{G})$ suggests the analogy with the basic example given by continuous functions on a compact group. In the quantum case however, there is no underlying space $\mathbb{G}$ and $C(\mathbb{G})$ is a non-abelian $\mathrm{C}^{*}$-algebra.

A fundamental result in the theory of compact quantum groups is the existence of a unique Haar state.

Theorem 1.3. Let $\mathbb{G}$ be a compact quantum group. There exists a unique state $h$ on $C(\mathbb{G})$ which satisfies $(\mathrm{id} \otimes h) \Delta(a)=h(a) 1=(h \otimes \mathrm{id}) \Delta(a)$ for all $a \in C(\mathbb{G})$. The state $h$ is called the Haar state of $\mathbb{G}$.

Another crucial set of results in the framework of compact quantum groups is the Peter-Weyl representation theory. 
Definition 1.4. A unitary representation $U$ of a compact quantum group $\mathbb{G}$ on a Hilbert space $H$ is a unitary element $U \in \mathrm{M}(\mathcal{K}(H) \otimes C(\mathbb{G}))$ satisfying

$$
(\mathrm{id} \otimes \Delta)(U)=U_{12} U_{13}
$$

Whenever $U^{1}$ and $U^{2}$ are unitary representations of $\mathbb{G}$ on the respective Hilbert spaces $H_{1}$ and $H_{2}$, we define

$$
\operatorname{Mor}\left(U^{1}, U^{2}\right):=\left\{T \in \mathrm{B}\left(H_{2}, H_{1}\right) \mid U_{1}(T \otimes 1)=(T \otimes 1) U_{2}\right\} .
$$

The elements of $\operatorname{Mor}\left(U^{1}, U^{2}\right)$ are called intertwiners. We use the notation $\operatorname{End}(U):=\operatorname{Mor}(U, U)$. A unitary representation $U$ is said to be irreducible if $\operatorname{End}(U)=\mathbb{C} 1$. If $\operatorname{Mor}\left(U^{1}, U^{2}\right)$ contains a unitary operator, the representations $U^{1}$ and $U^{2}$ are said to be unitarily equivalent.

We have the following essential result.

Theorem 1.5. Every irreducible representation of a compact quantum group is finite-dimensional. Every unitary representation is unitarily equivalent to a direct sum of irreducibles.

Because of this theorem, we almost exclusively deal with finite-dimensional representations. By choosing an orthonormal basis of the Hilbert space $H$, a finite-dimensional unitary representation of $\mathbb{G}$ can be considered as a unitary matrix $\left(U_{i j}\right)$ with entries in $C(\mathbb{G})$ and $(1.1)$ becomes

$$
\Delta\left(U_{i j}\right)=\sum_{k} U_{i k} \otimes U_{k j}
$$

The product in the $\mathrm{C}^{*}$-algebra $C(\mathbb{G})$ yields a tensor product on the level of unitary representations.

Definition 1.6. Let $U^{1}$ and $U^{2}$ be unitary representations of $\mathbb{G}$ on the respective Hilbert spaces $H_{1}$ and $H_{2}$. We define the tensor product

$$
U^{1} \odot U^{2}:=U_{13}^{1} U_{23}^{2} \in \mathrm{M}\left(\mathcal{K}\left(H_{1} \otimes H_{2}\right) \otimes C(\mathbb{G})\right) .
$$

Notation 1.7. Let $\mathbb{G}$ be a compact quantum group. We denote by $\operatorname{Irred}(\mathbb{G})$ the set of equivalence classes of irreducible unitary representations. We choose representatives $U^{x}$ on the Hilbert space $H_{x}$ for every $x \in \operatorname{Irred}(\mathbb{G})$. Whenever $x, y \in \operatorname{Irred}(\mathbb{G})$, we use $x \otimes y$ to denote the unitary representation $U^{x} \uparrow U^{y}$. The class of the trivial unitary representation is denoted by $\varepsilon$. We define the natural numbers mult $(x \otimes y, z) \operatorname{such}$ that

$$
x \otimes y \cong \bigoplus_{z \in \operatorname{Irred}(\mathbb{G})} \operatorname{mult}(x \otimes y, z) \cdot U^{z}
$$

The collection of natural numbers mult $(x \otimes y, z)$ are called the fusion rules of $\mathbb{G}$.

The set $\operatorname{Irred}(\mathbb{G})$ is equipped with a natural involution $x \mapsto \bar{x}$ such that $U^{\bar{x}}$ is the unique (up to unitary equivalence) irreducible unitary representation satisfying

$$
\operatorname{Mor}(x \otimes \bar{x}, \epsilon) \neq\{0\} \neq \operatorname{Mor}(\bar{x} \otimes x, \epsilon) .
$$

The unitary representation $U^{\bar{x}}$ is called the contragredient of $U^{x}$.

For every $x \in \operatorname{Irred}(\mathbb{G})$, we take non-zero elements $t_{x} \in \operatorname{Mor}(x \otimes \bar{x}, \epsilon)$ and $s_{x} \in \operatorname{Mor}(\bar{x} \otimes x, \varepsilon)$ satisfying $\left(t_{x}^{*} \otimes 1\right)\left(1 \otimes s_{x}\right)=1$. Write the antilinear map

$$
j_{x}: H_{x} \rightarrow H_{\bar{x}}: \xi \mapsto\left(\xi^{*} \otimes 1\right) t_{x}
$$

and define $Q_{x}:=j_{x}^{*} j_{x}$. We normalize $t_{x}$ in such a way that $\operatorname{Tr}\left(Q_{x}\right)=\operatorname{Tr}\left(Q_{x}^{-1}\right)$. This uniquely determines $Q_{x}$ and fixes $t_{x}, s_{x}$ up to a number of modulus 1 . Note that $t_{x}^{*} t_{x}=\operatorname{Tr}\left(Q_{x}\right)$.

Definition 1.8. For $x \in \operatorname{Irred}(\mathbb{G})$, the value $\operatorname{Tr}\left(Q_{x}\right)$ is called the quantum dimension of $x$ and denoted by $\operatorname{dim}_{q}(x)$. Note that $\operatorname{dim}_{q}(x) \geq \operatorname{dim}(x)$, with equality holding if and only if $Q_{x}=1$. 
The irreducible representations of $\mathbb{G}$ and the Haar state $h$ are connected by the orthogonality relations.

$$
(\mathrm{id} \otimes h)\left(U^{x}\left(\xi \eta^{*} \otimes 1\right)\left(U^{y}\right)^{*}\right)=\frac{\delta_{x, y} 1}{\operatorname{dim}_{q}(x)}\left\langle\eta, Q_{x} \xi\right\rangle \quad, \quad(\operatorname{id} \otimes h)\left(\left(U^{x}\right)^{*}\left(\xi \eta^{*} \otimes 1\right) U^{y}\right)=\frac{\delta_{x, y} 1}{\operatorname{dim}_{q}(x)}\left\langle\eta, Q_{x}^{-1} \xi\right\rangle,
$$

for $\xi \in H_{x}$ and $\eta \in H_{y}$.

Notation 1.9. Let $\mathbb{G}=(C(\mathbb{G}), \Delta)$ be a compact quantum group. We denote by $\mathcal{C}(\mathbb{G})$ the set of coefficients of finite dimensional representations of $\mathbb{G}$. Hence,

$$
\mathcal{C}(\mathbb{G})=\left\langle\left(\omega_{\xi, \eta} \otimes \mathrm{id}\right)\left(U^{x}\right) \mid x \in \operatorname{Irred}(\mathbb{G}), \xi, \eta \in H_{x}\right\rangle
$$

Then, $\mathcal{C}(\mathbb{G})$ is a unital dense *-subalgebra of $C(\mathbb{G})$. Restricting $\Delta$ to $\mathcal{C}(\mathbb{G}), \mathcal{C}(\mathbb{G})$ becomes a Hopf *-algebra. Also, for $x \in \operatorname{Irred}(\mathbb{G})$, denote by

$$
\mathcal{C}(\mathbb{G})_{x}=\left\langle\left(\omega_{\xi, \eta} \otimes \mathrm{id}\right)\left(U^{x}\right) \mid \xi, \eta \in H_{x}\right\rangle
$$

Definition 1.10. The reduced $C^{*}$-algebra $C_{r}(\mathbb{G})$ is defined as the norm closure of $\mathcal{C}(\mathbb{G})$ in the GNSrepresentation with respect to $h$. The universal $C^{*}$-algebra $C_{u}(\mathbb{G})$ is defined as the enveloping $\mathrm{C}^{*}$-algebra of $\mathcal{C}(\mathbb{G})$. The von Neumann algebra $L^{\infty}(\mathbb{G})$ is defined as the von Neumann algebra generated by $C_{r}(\mathbb{G})$.

A compact quantum group $\mathbb{G}$ is said to be co-amenable (and the discrete quantum group $\widehat{\mathbb{G}}$ is said to be amenable) if the homomorphism $C_{u}(\mathbb{G}) \rightarrow C_{r}(\mathbb{G})$ is an isomorphism.

Given an arbitrary compact quantum group $\mathbb{G}$, we have surjective homomorphisms $C_{u}(\mathbb{G}) \rightarrow C(\mathbb{G}) \rightarrow C_{r}(\mathbb{G})$, and we are only interested in $C_{r}(\mathbb{G})$ and $C_{u}(\mathbb{G})$. Note that if $\mathbb{G}$ is the dual of a discrete group $\Gamma$, we have $C_{r}(\mathbb{G})=C_{r}^{*}(\Gamma)$ and $C_{u}(\mathbb{G})=C^{*}(\Gamma)$.

Proposition 1.11. The Haar state $h$ is a KMS-state on both $C_{r}(\mathbb{G})$ and $C_{u}(\mathbb{G})$ and the modular group is determined by

$$
\left(\mathrm{id} \otimes \sigma_{t}^{h}\right)\left(U^{x}\right)=\left(Q_{x}^{i t} \otimes 1\right) U^{x}\left(Q_{x}^{i t} \otimes 1\right)
$$

for every $x \in \operatorname{Irred}(\mathbb{G})$.

\section{Discrete quantum groups and duality}

A discrete quantum group is defined as the dual of a compact quantum group by putting together all irreducible representations.

Definition 1.12. Let $\mathbb{G}$ be a compact quantum group. We define the dual (discrete) quantum group $\widehat{\mathbb{G}}$ as follows.

$$
c_{0}(\widehat{\mathbb{G}})=\bigoplus_{x \in \operatorname{Irred}(\mathbb{G})} \mathrm{B}\left(H_{x}\right), \quad \ell^{\infty}(\widehat{\mathbb{G}})=\prod_{x \in \operatorname{Irred}(\mathbb{G})} \mathrm{B}\left(H_{x}\right) .
$$

We denote the minimal central projections of $\ell^{\infty}(\widehat{\mathbb{G}})$ by $p_{x}, x \in \operatorname{Irred}(\mathbb{G})$. We have a natural unitary $\mathbb{V} \in \mathrm{M}\left(c_{0}(\widehat{\mathbb{G}}) \otimes C(\mathbb{G})\right)$ given by

$$
\mathbb{V}=\bigoplus_{x \in \operatorname{Irred}(\mathbb{G})} U^{x}
$$

This unitary $\mathbb{V}$ implements the duality between $\mathbb{G}$ and $\widehat{\mathbb{G}}$. We have a natural comultiplication

$$
\hat{\Delta}: \ell^{\infty}(\widehat{\mathbb{G}}) \rightarrow \ell^{\infty}(\widehat{\mathbb{G}}) \otimes \ell^{\infty}(\widehat{\mathbb{G}}):(\hat{\Delta} \otimes \mathrm{id})(\mathbb{V})=\mathbb{V}_{13} \mathbb{V}_{23} .
$$

One can deduce from this the following equivalent way to define the comultiplication on $\ell^{\infty}(\widehat{\mathbb{G}})$.

$$
\hat{\Delta}(a) S=S a \text { for all } a \in \ell^{\infty}(\widehat{\mathbb{G}}), S \in \operatorname{Mor}(y \otimes z, x) .
$$

The notation introduced above is aimed to suggest the basic example where $\mathbb{G}$ is the dual of a discrete group $\Gamma$, given by $C(\mathbb{G})=C^{*}(\Gamma)$ and $\Delta\left(\lambda_{x}\right)=\lambda_{x} \otimes \lambda_{x}$ for all $x \in \Gamma$. The map $x \mapsto \lambda_{x}$ yields an identification of $\Gamma$ and $\operatorname{Irred}(\mathbb{G})$ and then, $\ell^{\infty}(\widehat{\mathbb{G}})=\ell^{\infty}(\Gamma)$. 
Remark 1.13. It is possible to give an intrinsic definition of a discrete quantum group. This was already implicitly clear in Woronowicz' Tannaka-Krein theorem and was explicitly done in [8, 17]. For our purposes, it is most convenient to take the compact quantum group as a starting point: indeed, most examples of concrete discrete quantum groups (and that are not discrete groups) are defined as the dual of a compact quantum group.

The discrete quantum group $\ell^{\infty}(\widehat{\mathbb{G}})$ comes equipped with a natural modular structure.

Notation 1.14. We have canonically defined states $\varphi_{x}$ and $\psi_{x}$ on $\mathrm{B}\left(H_{x}\right)$ related to $(1.2)$ as follows.

$$
\begin{aligned}
& \psi_{x}(A)=\frac{1}{\operatorname{dim}_{q}(x)} t_{x}^{*}(A \otimes 1) t_{x}=\frac{\operatorname{Tr}\left(Q_{x} A\right)}{\operatorname{Tr}\left(Q_{x}\right)}=(\mathrm{id} \otimes h)\left(U^{x}(A \otimes 1)\left(U^{x}\right)^{*}\right) \quad \text { and } \\
& \varphi_{x}(A)=\frac{1}{\operatorname{dim}_{q}(x)} t_{\bar{x}}^{*}(1 \otimes A) t_{\bar{x}}=\frac{\operatorname{Tr}\left(Q_{x}^{-1} A\right)}{\operatorname{Tr}\left(Q_{x}^{-1}\right)}=(\mathrm{id} \otimes h)\left(\left(U^{x}\right)^{*}(A \otimes 1) U^{x}\right),
\end{aligned}
$$

for all $A \in \mathrm{B}\left(H_{x}\right)$.

Remark 1.15. The states $\varphi_{x}$ and $\psi_{x}$ are significant, since they provide a formula for the Haar weights on $\ell^{\infty}(\widehat{\mathbb{G}})$. The left Haar weight is given by $\sum_{x \in \operatorname{Irred}(\mathbb{G})} \operatorname{dim}_{q}(x)^{2} \psi_{x}$, and the right Haar weight is given by $\sum_{x \in \operatorname{Irred}(\mathbb{G})} \operatorname{dim}_{q}(x)^{2} \varphi_{x}$.

\section{Examples: the universal orthogonal compact quantum groups}

This class of compact quantum groups was introduced by Wang and Van Daele in [18] and studied by Banica in [1].

Definition 1.16. Let $F \in \mathrm{GL}(n, \mathbb{C})$ satisfying $F \bar{F}= \pm 1$. We define the compact quantum group $\mathbb{G}=A_{o}(F)$ as follows.

- $C(\mathbb{G})$ is the universal $\mathrm{C}^{*}$-algebra with generators $\left(U_{i j}\right)$ and relations making $U=\left(U_{i j}\right)$ a unitary element of $\mathrm{M}_{n}(\mathbb{C}) \otimes C(\mathbb{G})$ and satisfying $U=F \bar{U} F^{-1}$, where $(\bar{U})_{i j}=\left(U_{i j}\right)^{*}$.

- $\Delta\left(U_{i j}\right)=\sum_{k} U_{i k} \otimes U_{k j}$.

In these examples, the unitary matrix $U$ is a representation, called the fundamental representation. The definition of $\mathbb{G}=A_{o}(F)$ makes sense without the requirement $F \bar{F}= \pm 1$, but the fundamental representation is irreducible if and only if $F \bar{F} \in \mathbb{R} 1$. We then normalize such that $F \bar{F}= \pm 1$.

Remark 1.17. It is easy to classify the quantum groups $A_{o}(F)$. For $F_{1}, F_{2} \in \operatorname{GL}(n, \mathbb{C})$ with $F_{i} \bar{F}_{i}= \pm 1$, we write $F_{1} \sim F_{2}$ if there exists a unitary matrix $v$ such that $F_{1}=v F_{2} v^{t}$, where $v^{t}$ is the transpose of $v$. Then, $A_{o}\left(F_{1}\right) \cong A_{o}\left(F_{2}\right)$ if and only if $F_{1} \sim F_{2}$. It follows that the $A_{o}(F)$ are classified up to isomorphism by $n$, the sign $F \bar{F}$ and the eigenvalue list of $F^{*} F$ (see e.g. Section 5 of [6] where an explicit fundamental domain for the relation $\sim$ is described).

If $F \in \mathrm{GL}(2, \mathbb{C})$, we get up to equivalence, the matrices

$$
F_{q}=\left(\begin{array}{cc}
0 & |q|^{1 / 2} \\
-(\operatorname{sgn} q)|q|^{-1 / 2} & 0
\end{array}\right)
$$

for $q \in[-1,1], q \neq 0$, with corresponding quantum groups $A_{o}\left(F_{q}\right) \cong \mathrm{SU}_{q}(2)$, see [20].

The following result has been proved by Banica [1]. It tells us that the compact quantum groups $A_{o}(F)$ have the same fusion rules as the group $\mathrm{SU}(2)$.

Theorem 1.18. Let $F \in \mathrm{GL}(n, \mathbb{C})$ and $F \bar{F}= \pm 1$. Let $\mathbb{G}=A_{o}(F)$. Then $\operatorname{Irred}(\mathbb{G})$ can be identified with $\mathbb{N}$ in such a way that

$$
x \otimes y \cong|x-y| \oplus(|x-y|+2) \oplus \cdots \oplus(x+y),
$$

for all $x, y \in \mathbb{N}$. 


\section{Actions of quantum groups and spectral subspaces}

Definition 1.19. Let $B$ be a unital $C^{*}$-algebra. A (right) action of $\mathbb{G}$ on $B$ is a unital *-homomorphism $\delta: B \rightarrow B \otimes C(\mathbb{G})$ satisfying

$$
(\delta \otimes \mathrm{id}) \delta=(\mathrm{id} \otimes \Delta) \delta \quad \text { and } \quad[\delta(B)(1 \otimes C(\mathbb{G}))]=B \otimes C(\mathbb{G}) .
$$

The action $\delta$ is said to be ergodic if the fixed point algebra $B^{\delta}:=\{x \in B \mid \delta(x)=x \otimes 1\}$ equals $\mathbb{C} 1$. In that case, $B$ admits a unique invariant state $\omega$ given by $\omega(b) 1=($ id $\otimes h) \delta(b)$.

Definition 1.20. Let $\delta: B \rightarrow B \otimes C(\mathbb{G})$ be an action of the compact quantum group $\mathbb{G}$ on the unital $\mathrm{C}^{*}$-algebra $B$.

- For every $x \in \operatorname{Irred}(\mathbb{G})$, the spectral subspace $B_{x}$ is defined as the linear subspace $B_{x} \subset B$ given by

$$
B_{x}:=\left\{b \in B \mid \delta(b) \in B \otimes \mathcal{C}(\mathbb{G})_{x}\right\}
$$

Note that $\delta: B_{x} \rightarrow B_{x} \otimes \mathcal{C}(\mathbb{G})_{x}$ and that $B_{x}^{*}=B_{\bar{x}}$.

- We define $\mathcal{B}$ as the linear span of the $B_{x}, x \in \operatorname{Irred}(\mathbb{G})$. Then, $\mathcal{B}$ is a dense ${ }^{*}$-subalgebra of $B$.

- The action $\delta$ is said to be universal if $B$ is the universal enveloping $\mathrm{C}^{*}$-algebra of $\mathcal{B}$. It is said to be reduced if the conditional expectation (id $\otimes h) \delta$ of $B$ on $B^{\delta}$ is faithful.

- If $\delta$ is ergodic, $B_{x}$ is finite dimensional and its dimension is of the form $\operatorname{dim} H_{x} \cdot \operatorname{mult}(x, \delta)$, where mult $(x, \delta)$ is called the multiplicity of $x$ in $\delta$.

Remark 1.21. If $\mathbb{G}$ is co-amenable, $C(\mathbb{G})$ has a bounded co-unit and a faithful Haar state. Hence, any action of $\mathbb{G}$ is both universal and reduced.

Actions on von Neumann algebras are defined as follows.

Definition 1.22. A right action of a compact (resp. discrete) quantum group $\mathbb{G}$ (resp. $\widehat{\mathbb{G}}$ ) on a von Neumann algebra $N$ is an injective normal unital *-homomorphism

$$
\delta: N \rightarrow N \otimes L^{\infty}(\mathbb{G}) \quad \text { resp. } \quad \delta: N \rightarrow N \otimes \ell^{\infty}(\widehat{\mathbb{G}})
$$

satisfying $(\delta \otimes \mathrm{id}) \delta=(\mathrm{id} \otimes \Delta) \delta$, resp. $(\delta \otimes \mathrm{id}) \delta=(\mathrm{id} \otimes \hat{\Delta}) \delta$.

\section{The Poisson boundary of a discrete quantum group}

We give a brief survey of Izumi's theory of Poisson boundaries for discrete quantum groups and his computation of the Poisson boundary for the dual of $\mathrm{SU}_{q}(2)$, see [10]. We state as well some of the results of [11] that are useful in the rest of the paper.

\section{General results}

Fix a discrete quantum group $\widehat{\mathbb{G}}$.

Notation 2.1. For every normal state $\phi \in \ell^{\infty}(\widehat{\mathbb{G}})$, we define the convolution operator

$$
P_{\phi}: \ell^{\infty}(\widehat{\mathbb{G}}) \rightarrow \ell^{\infty}(\widehat{\mathbb{G}}): P_{\phi}(a)=(\mathrm{id} \otimes \phi) \widehat{\Delta}(a)
$$


We are only interested in special states $\phi \in \ell^{\infty}(\widehat{\mathbb{G}})$, motivated by Proposition 2.2 below. For every probability measure $\mu$ on $\operatorname{Irred}(\mathbb{G})$, we set

$$
\psi_{\mu}=\sum_{x \in \operatorname{Irred}(\mathbb{G})} \mu(x) \psi_{x} \text { and } P_{\mu}:=P_{\psi_{\mu}} .
$$

Recall that the states $\psi_{x}$ are defined in 1.14. Note that we have a convolution product $\mu * \nu$ on the measures on $\operatorname{Irred}(G)$, such that $\psi_{\mu * \nu}=\left(\psi_{\mu} \otimes \psi_{\nu}\right) \hat{\Delta}$.

Proposition 2.2. Let $\phi$ be a normal state on $\ell^{\infty}(\widehat{\mathbb{G}})$. Then the following conditions are equivalent.

- $\phi$ has the form $\psi_{\mu}$ from some probability measure $\mu$ on $\operatorname{Irred}(\mathbb{G})$.

- The Markov operator $P_{\phi}$ preserves the center of $\ell^{\infty}(\widehat{\mathbb{G}})$.

- $\phi$ is invariant under the adjoint action $\alpha_{\mathbb{G}}: \ell^{\infty}(\widehat{\mathbb{G}}) \rightarrow \ell^{\infty}(\widehat{\mathbb{G}}) \otimes L^{\infty}(\mathbb{G}): a \mapsto \mathbb{V}(a \otimes 1) \mathbb{V}^{*}$.

Definition 2.3 ([10], Section 2.5). Let $\mu$ be a probability measure on $\operatorname{Irred}(\mathbb{G})$. Set

$$
H^{\infty}(\widehat{\mathbb{G}}, \mu)=\left\{a \in \ell^{\infty}(\widehat{\mathbb{G}}) \mid P_{\mu}(a)=a\right\} .
$$

Equipped with the product defined by

$$
a \cdot b:=s^{*}-\lim _{n \rightarrow \infty} \frac{1}{n} \sum_{k=1}^{n} P_{\mu}^{k}(a b)
$$

the space $H^{\infty}(\widehat{\mathbb{G}}, \mu)$ becomes a von Neumann algebra that we call the Poisson boundary of $\widehat{\mathbb{G}}$ with respect to $\mu$.

Terminology 2.4. A probability measure $\mu$ on $\operatorname{Irred}(\mathbb{G})$ is called generating if there exists, for every $x \in \operatorname{Irred}(\mathbb{G})$, an $n \geq 1$ such that $\mu^{* n}(x) \neq 0$.

In all the results below, the probability measure $\mu$ is assumed to be generating. In that case, it is not hard to concretely realize $H^{\infty}(\widehat{\mathbb{G}}, \mu)$ as a von Neumann algebra. Indeed,

$$
\pi_{\infty}: H^{\infty}(\widehat{\mathbb{G}}, \mu) \rightarrow \bigotimes_{n=0}^{\infty}\left(\ell^{\infty}(\widehat{\mathbb{G}}), \psi_{\mu}\right): \pi_{\infty}(a)=s^{*}-\lim _{n \rightarrow \infty} \pi_{n}(a) \quad \text { where } \pi_{n}:=\hat{\Delta}^{(n)}: \ell^{\infty}(\widehat{\mathbb{G}}) \rightarrow \bigotimes_{k=0}^{n-1} \ell^{\infty}(\widehat{\mathbb{G}}) .
$$

Moreover, the product becomes $a \cdot b=s^{*}-\lim _{n \rightarrow \infty} P_{\mu}^{n}(a b)$.

The restriction of the co-unit $\widehat{\varepsilon}$ yields a state on $H^{\infty}(\widehat{\mathbb{G}}, \mu)$, called the harmonic state. It is clear that this state is faithful when $\mu$ is generating.

Definition 2.5. Let $\mu$ be a generating measure on $\operatorname{Irred}(\mathbb{G})$. The Poisson boundary $H^{\infty}(\widehat{\mathbb{G}}, \mu)$ comes equipped with two natural actions, one of $\mathbb{G}$ and one of $\widehat{\mathbb{G}}$ :

$$
\begin{aligned}
& \alpha_{\mathbb{G}}: H^{\infty}(\widehat{\mathbb{G}}, \mu) \rightarrow H^{\infty}(\widehat{\mathbb{G}}, \mu) \otimes L^{\infty}(\mathbb{G}): \alpha_{\mathbb{G}}(a)=\mathbb{V}(a \otimes 1) \mathbb{V}^{*}, \\
& \alpha_{\widehat{\mathbb{G}}}: H^{\infty}(\widehat{\mathbb{G}}, \mu) \rightarrow \ell^{\infty}(\widehat{\mathbb{G}}) \otimes H^{\infty}(\widehat{\mathbb{G}}, \mu): \alpha_{\widehat{\mathbb{G}}}(a)=\hat{\Delta}(a) .
\end{aligned}
$$

Note that $\alpha_{\mathbb{G}}$ is the restriction of the adjoint action of $\mathbb{G}$ on $\ell^{\infty}(\widehat{\mathbb{G}})$, while $\alpha_{\widehat{\mathbb{G}}}$ is nothing else than the restriction of the comultiplication. The actions $\alpha_{\mathbb{G}}$ and $\alpha_{\widehat{\mathbb{G}}}$ are well defined because of the following equivariance formulae:

$$
\left(\mathrm{id} \otimes P_{\mu}\right)(\widehat{\Delta}(a))=\widehat{\Delta}\left(P_{\mu}(a)\right) \quad \text { and } \quad\left(P_{\mu} \otimes \mathrm{id}\right)\left(\alpha_{\mathbb{G}}(a)\right)=\alpha_{\mathbb{G}}\left(P_{\mu}(a)\right) .
$$

When $\widehat{\mathbb{G}}$ is a discrete group, the action $\alpha_{\mathbb{G}}$ is the trivial action on $\ell^{\infty}(\widehat{\mathbb{G}})$. In general, the fixed point algebra of $\alpha_{\mathbb{G}}$ is precisely the algebra of central harmonic elements $Z\left(\ell^{\infty}(\widehat{\mathbb{G}})\right) \cap H^{\infty}(\widehat{\mathbb{G}}, \mu)$. Since the Markov operator 
$P_{\mu}$ preserves the center $Z\left(\ell^{\infty}(\widehat{\mathbb{G}})\right)$, the commutative von Neumann algebra $Z\left(\ell^{\infty}(\widehat{\mathbb{G}})\right) \cap H^{\infty}(\widehat{\mathbb{G}}, \mu)$ with state $\widehat{\varepsilon}$, is exactly the Poisson boundary for the random walk on $\operatorname{Irred}(\mathbb{G})$ with transition probabilities $p(x, y)$ and $n$-step transition probabilities $p_{n}(x, y)$ given by

$$
p_{x} p(x, y)=p_{x} P_{\mu}\left(p_{y}\right), \quad p_{x} p_{n}(x, y)=p_{x} P_{\mu}^{n}\left(p_{y}\right) .
$$

Note that $p_{n}(e, y)=\mu^{* n}(y)=\psi_{\mu}^{* n}\left(p_{y}\right)$.

So, the action $\alpha_{\mathbb{G}}$ is ergodic if and only if there are no non-trivial central harmonic elements. This occurs if the fusion algebra of $\mathbb{G}$ is commutative. Much more can be said in that case. We record the following results for future use.

Proposition 2.6. Suppose that the fusion algebra of $\mathbb{G}$ is commutative (i.e. $\operatorname{mult}(y \otimes z, x)=\operatorname{mult}(z \otimes y, x)$ for every $x, y, z \in \operatorname{Irred}(\mathbb{G})$ ) and let $\mu$ be a generating probability measure on $\operatorname{Irred}(\mathbb{G})$.

- (Cor. 3.5 in [9] and Cor. 3.2 in [11]) There are no non-trivial central harmonic elements, i.e.

$$
Z\left(\ell^{\infty}(\widehat{\mathbb{G}})\right) \cap H^{\infty}(\widehat{\mathbb{G}}, \mu)=\mathbb{C} 1 .
$$

- (Prop. 1.1 in [11]) The Poisson boundary does not depend on the choice of generating measure:

$$
H^{\infty}(\widehat{\mathbb{G}}, \mu)=\left\{a \in \ell^{\infty}(\widehat{\mathbb{G}}) \mid P_{x}(a)=a \quad \text { for all } x \in \operatorname{Irred}(\mathbb{G})\right\}
$$

- (Cor. 3.5 in [11]) Using the notation of 1.20, we have

$$
\operatorname{mult}\left(x, \alpha_{\mathbb{G}}\right) \leq \sup \{\operatorname{mult}(\bar{y} \otimes y, x) \mid y \in \operatorname{Irred}(\mathbb{G})\},
$$

for all $x \in \operatorname{Irred}(\mathbb{G})$.

\section{The Poisson boundary of $\widehat{S U_{q}(2)}$}

Let $\mathbb{G}=\mathrm{SU}_{q}(2)$ for $-1<q<0$ or $0<q<1$. In [10], Izumi identified the Poisson boundary of $\widehat{\mathbb{G}}$ with the Podles sphere. Since this result is needed in our identification of the Poisson boundary of the dual of $A_{o}(F)$, we briefly recall it here. We also refer to [11] for an easier approach to the computation of the Poisson boundary of the dual of $\mathrm{SU}_{q}(2)$.

We first record the following general result.

Proposition 2.7 (Lemma 3.8 in [10]). Let $\mathbb{G}$ be a compact quantum group. Defining

$$
\Phi: L^{\infty}(\mathbb{G}) \rightarrow \ell^{\infty}(\widehat{\mathbb{G}}): \Phi(a)=(\mathrm{id} \otimes h)\left(\mathbb{V}^{*}(1 \otimes a) \mathbb{V}\right)
$$

the image of $\Phi$ is contained in $H^{\infty}(\widehat{\mathbb{G}}, \mu)$ for any probability measure $\mu$ on $\operatorname{Irred}(\mathbb{G})$.

For the rest of this section, fix $q \in]-1,1\left[, q \neq 0\right.$ and set $\mathbb{G}=\mathrm{SU}_{q}(2)$ (see [20]). Recall that $C\left(S U_{q}(2)\right.$ ) is generated by the coefficients of the unitary operator $U=\left(\begin{array}{cc}\alpha & -q \gamma^{*} \\ \gamma & \alpha^{*}\end{array}\right)$ and that $\Delta$ is defined such that $U$ is a unitary representation of $\mathrm{SU}_{q}(2)$, called the fundamental representation.

Consider the natural homomorphism

$$
\pi_{S^{1}}: C\left(\mathrm{SU}_{q}(2)\right) \rightarrow C\left(S^{1}\right): \pi_{S^{1}}(\alpha)=z \quad \text { and } \quad \pi_{S^{1}}(\gamma)=0
$$

So, $\Delta_{S^{1}} \pi_{S^{1}}=\left(\pi_{S^{1}} \otimes \pi_{S^{1}}\right) \Delta$, and we can consider $\pi_{S^{1}}$ as an embedding of the circle $S^{1}$ into $\mathrm{SU}_{q}(2)$.

Define

$$
C\left(S^{1} \backslash \mathrm{SU}_{q}(2)\right):=\left\{a \in \mathrm{SU}_{q}(2) \mid\left(\pi_{S^{1}} \otimes 1\right) \Delta(a)=1 \otimes a\right\}
$$

This homogeneous space is just the fixed point algebra of the action

$$
\Delta_{S^{1}}:=\left(\pi_{S^{1}} \otimes \mathrm{id}\right) \Delta: C\left(S U_{q}(2)\right) \rightarrow C\left(S^{1}\right) \otimes C\left(S U_{q}(2)\right)
$$


and is called the Podleś sphere [15].

Observe that the restriction of the comultiplication yields a right action $\beta_{\mathbb{G}}$ of $\mathbb{G}=\mathrm{SU}_{q}(2)$ on $L^{\infty}\left(S^{1} \backslash \mathbb{G}\right)$. Moreover, the restriction of the adjoint action

$$
\beta_{\widehat{\mathbb{G}}}: L^{\infty}(\mathbb{G}) \rightarrow \ell^{\infty}(\widehat{\mathbb{G}}) \otimes L^{\infty}(\mathbb{G}): \beta_{\widehat{\mathbb{G}}}(a)=\mathbb{V}^{*}(1 \otimes a) \mathbb{V}
$$

yields a left action of $\widehat{\mathbb{G}}$ on $C\left(S^{1} \backslash \mathbb{G}\right)$.

Theorem 2.8 (Th. 5.10 in $[10])$. Let $\mathbb{G}=\mathrm{SU}_{q}(2)(q \neq \pm 1)$ and let $\mu$ be a generating probability measure on $\operatorname{Irred}(\mathbb{G})$. The restriction of the completely positive map $\Phi$ (see Proposition 2.7) to $L^{\infty}\left(S^{1} \backslash \mathbb{G}\right.$ ) is a *-isomorphism $\Phi_{0}$ between the Podleś sphere and the Poisson boundary of $\widehat{\mathbb{G}}$.

The ${ }^{*}$-isomorphism $\Phi_{0}$ intertwines the actions $\beta_{\mathbb{G}}, \beta_{\widehat{\mathbb{G}}}$ on $L^{\infty}\left(S^{1} \backslash \mathbb{G}\right)$ with the actions $\alpha_{\mathbb{G}}, \alpha_{\widehat{\mathbb{G}}}$ on $H^{\infty}(\widehat{\mathbb{G}}, \mu)$ defined in 2.5. Finally, $\Phi_{0}$ intertwines the restriction of the Haar state with the harmonic state $\widehat{\varepsilon}$.

\section{The Martin boundary of a discrete quantum group}

The Martin boundary and the Martin compactification of a discrete quantum group have been defined by Neshveyev and Tuset in [14]. Fix a discrete quantum group $\widehat{\mathbb{G}}$ and a probability measure $\mu$ on $\operatorname{Irred}(\mathbb{G})$. We have an associated Markov operator $P_{\mu}$ and a classical random walk on $\operatorname{Irred}(\mathbb{G})$ with $n$-step transition probabilities given by (2.1).

Definition 3.1. The probability measure $\mu$ on $\operatorname{Irred}(\mathbb{G})$ is said to be transient if $\sum_{n=0}^{\infty} p_{n}(x, y)<\infty$ for all $x, y \in \operatorname{Irred}(\mathbb{G})$.

We suppose throughout that $\mu$ is a generating measure and that $\mu$ is transient.

Denote by $c_{c}(\widehat{\mathbb{G}}) \subset c_{0}(\widehat{\mathbb{G}})$ the algebraic direct sum of the algebras $\mathrm{B}\left(H_{x}\right)$. We define, for $a \in c_{c}(\widehat{\mathbb{G}})$,

$$
G_{\mu}(a)=\sum_{n=0}^{\infty} P_{\mu}^{n}(a) .
$$

Observe that usually $G_{\mu}(a)$ is unbounded, but it makes sense in the multiplier algebra of $c_{c}(\widehat{\mathbb{G}})$, i.e. $G_{\mu}(a) p_{x} \in$ $\mathrm{B}\left(H_{x}\right)$ makes sense for every $x \in \operatorname{Irred}(\mathbb{G})$ because $\mu$ is transient. Moreover, $G_{\mu}\left(p_{\epsilon}\right)$ is strictly positive and central. This allows to define the Martin kernel as follows.

Whenever $\mu$ is a measure on $\operatorname{Irred}(\mathbb{G})$, we use the notation $\bar{\mu}$ to denote the measure given by $\bar{\mu}(x)=\mu(\bar{x})$.

Definition 3.2 (Defs. 3.1 and 3.2 in [14]). Define

$$
K_{\mu}: c_{c}(\widehat{\mathbb{G}}) \rightarrow \ell^{\infty}(\widehat{\mathbb{G}}): K_{\mu}(a)=G_{\mu}(a) G_{\mu}\left(p_{\epsilon}\right)^{-1} .
$$

Define the Martin compactification $\widetilde{A}_{\mu}$ as the $\mathrm{C}^{*}$-subalgebra of $\ell^{\infty}(\widehat{\mathbb{G}})$ generated by $K_{\bar{\mu}}\left(c_{c}(\widehat{\mathbb{G}})\right)$ and $c_{0}(\widehat{\mathbb{G}})$. Define the Martin boundary $A_{\mu}$ as the quotient $\widetilde{A}_{\mu} / c_{0}(\widehat{\mathbb{G}})$.

By Theorem 3.5 in [14], the adjoint action $\alpha_{\mathbb{G}}$ and the comultiplication $\hat{\Delta}$ define, by restriction and passage to the quotient, the following actions on the Martin boundary.

$$
\gamma_{\mathbb{G}}: A_{\mu} \rightarrow A_{\mu} \otimes C(\mathbb{G}) \quad \text { and } \quad \gamma_{\widehat{\mathbb{G}}}: A_{\mu} \rightarrow \mathrm{M}\left(c_{0}(\widehat{\mathbb{G}}) \otimes A_{\mu}\right) .
$$

\section{Monoidal equivalence}

A crucial tool in the computation of the Poisson boundary for $\widehat{\mathbb{G}}$ when $\mathbb{G}=A_{o}(F)$ is the monoidal equivalence of $A_{o}(F)$ and $\mathrm{SU}_{q}(2)$ for the appropriate value of $q$. This notion was introduced in [6] and is reviewed in this section. 
Definition 4.1 (Def. 3.1 in $[6])$. Two compact quantum groups $\mathbb{G}_{1}=\left(C\left(\mathbb{G}_{1}\right), \Delta\right)$ and $\mathbb{G}_{2}=\left(C\left(\mathbb{G}_{2}\right), \Delta_{2}\right)$ are said to be monoidally equivalent if there exists a bijection $\varphi: \operatorname{Irred}\left(\mathbb{G}_{1}\right) \rightarrow \operatorname{Irred}\left(\mathbb{G}_{2}\right) \operatorname{satisfying} \varphi(\varepsilon)=\varepsilon$, together with linear isomorphisms

$$
\varphi: \operatorname{Mor}\left(x_{1} \otimes \cdots \otimes x_{r}, y_{1} \otimes \cdots \otimes y_{k}\right) \rightarrow \operatorname{Mor}\left(\varphi\left(x_{1}\right) \otimes \cdots \otimes \varphi\left(x_{r}\right), \varphi\left(y_{1}\right) \otimes \cdots \otimes \varphi\left(y_{k}\right)\right)
$$

satisfying the following conditions:

$$
\begin{aligned}
\varphi(1) & =1 & \varphi(S \otimes T) & =\varphi(S) \otimes \varphi(T) \\
\varphi\left(S^{*}\right) & =\varphi(S)^{*} & \varphi(S T) & =\varphi(S) \varphi(T)
\end{aligned}
$$

whenever the formulas make sense. In the first formula, we consider $1 \in \operatorname{Mor}(x, x)=\operatorname{Mor}(x \otimes \varepsilon, x)=$ $\operatorname{Mor}(\varepsilon \otimes x, x)$. Such a collection of maps $\varphi$ is called a monoidal equivalence between $\mathbb{G}_{1}$ and $\mathbb{G}_{2}$.

By Theorem 3.9 and Proposition 3.13 of [6], we have the following.

Theorem 4.2. Let $\varphi$ be a monoidal equivalence between compact quantum groups $\mathbb{G}_{1}$ and $\mathbb{G}_{2}$.

- There exist a unique unital ${ }^{*}$-algebra $\mathcal{B}$ equipped with a faithful state $\omega$ and unitary elements $X^{x} \in$ $\mathrm{B}\left(H_{x}, H_{\varphi(x)}\right) \otimes \mathcal{B}$ for all $x \in \widehat{\mathbb{G}}_{1}$, satisfying

1. $X_{13}^{y} X_{23}^{z}(S \otimes 1)=(\varphi(S) \otimes 1) X^{x} \quad$ for all $S \in \operatorname{Mor}(y \otimes z, x)$,

2. the matrix coefficients of the $X^{x}$ form a linear basis of $\mathcal{B}$,

3. $(\mathrm{id} \otimes \omega)\left(X^{x}\right)=0 \quad$ if $\quad x \neq \epsilon$.

- There exists unique commuting ergodic actions $\delta_{1}: \mathcal{B} \rightarrow \mathcal{B} \otimes C\left(\mathbb{G}_{1}\right)$ and $\delta_{2}: \mathcal{B} \rightarrow C\left(\mathbb{G}_{2}\right) \otimes \mathcal{B}$ satisfying

$$
\left(\mathrm{id} \otimes \delta_{1}\right)\left(X^{x}\right)=X_{12}^{x} U_{13}^{x} \quad \text { and } \quad\left(\mathrm{id} \otimes \delta_{2}\right)\left(X^{x}\right)=U_{12}^{\varphi(x)} X_{13}^{x}
$$

for all $x \in \operatorname{Irred}\left(\mathbb{G}_{1}\right)$.

- The state $\omega$ is invariant under $\delta_{1}$ and $\delta_{2}$. Denoting by $B_{r}$ the $C^{*}$-algebra generated by $\mathcal{B}$ in the $G N S$ representation associated with $\omega$ and denoting by $B_{u}$ the universal enveloping $C^{*}$-algebra of $\mathcal{B}$, the actions $\delta_{1}, \delta_{2}$ admit unique extensions to actions on $B_{r}$ and $B_{u}$.

Note that in the case $\mathbb{G}=\mathbb{G}_{1}=\mathbb{G}_{2}$ and $\varphi$ the identity map, we have $\mathcal{B}=\mathcal{C}(\mathbb{G})$ and $X^{x}=U^{x}$ for every $x \in \operatorname{Irred}(\mathbb{G})$. The following unitary operator generalizes (1.3).

$$
\mathbb{X}:=\bigoplus_{x \in \operatorname{Irred}(\mathbb{G})} X^{x} \quad \text { where } \quad \mathbb{X} \in \prod_{x \in \operatorname{Irred}(\mathbb{G})}\left(\mathrm{B}\left(H_{x}, H_{\varphi(x)}\right) \otimes B\right)
$$

Proposition 4.3. The invariant state $\omega$ is a KMS state on $B_{r}$ and $B_{u}$ and its modular group is determined by

$$
\left(\mathrm{id} \otimes \sigma_{t}^{\omega}\right)\left(X^{x}\right)=\left(Q_{\varphi(x)}^{i t} \otimes 1\right) X^{x}\left(Q_{x}^{i t} \otimes 1\right)
$$

for every $x \in \operatorname{Irred}\left(\mathbb{G}_{1}\right)$.

Remark 4.4. Define $B_{x}:=\left\langle\left(\omega_{\xi, \eta} \otimes \mathrm{id}\right)\left(X^{x}\right) \mid \xi \in H_{\varphi(x)}, \eta \in H_{x}\right\rangle$. Then, as a vector space

$$
\mathcal{B}=\bigoplus_{x \in \operatorname{Irred}(\mathbb{G})} B_{x}
$$

Moreover, the $B_{x}$ are exactly the spectral subspaces of $\delta_{1}$ and $\delta_{2}$, while $\mathcal{B}$ is exactly the dense ${ }^{*}$-algebra given by Definition 1.20. 
The orthogonality relations (1.2) generalize and take the following form.

$$
\begin{aligned}
(\mathrm{id} \otimes \omega)\left(X^{x}\left(\xi_{1} \eta_{1}^{*} \otimes 1\right)\left(X^{y}\right)^{*}\right) & =\frac{\delta_{x, y} 1}{\operatorname{dim}_{q}(x)}\left\langle\eta_{1}, Q_{x} \xi_{1}\right\rangle, \\
(\mathrm{id} \otimes \omega)\left(\left(X^{x}\right)^{*}\left(\left(\xi_{2} \eta_{2}^{*} \otimes 1\right) X^{y}\right)\right. & =\frac{\delta_{x, y} 1}{\operatorname{dim}_{q}(x)}\left\langle\eta_{2}, Q_{\varphi(x)}^{-1} \xi_{2}\right\rangle,
\end{aligned}
$$

for $\xi_{1} \in H_{x}, \eta_{1} \in H_{y}, \xi_{2} \in H_{\varphi(x)}$ and $\eta_{2} \in H_{\varphi(y)}$.

We turn to the case where $\mathbb{G}_{1}=A_{o}\left(F_{1}\right)$ and $\mathbb{G}_{2}=A_{o}\left(F_{2}\right)$, which will be needed in this article.

Theorem 4.5 (Thms. 5.3 and 5.4 in $[6]$ ). Let $F_{1} \in M_{n_{1}}(\mathbb{C})$ and $F_{2} \in M_{n_{2}}(\mathbb{C})$ such that $F_{1} \bar{F}_{1}= \pm 1$ and $F_{2} \bar{F}_{2}= \pm 1$.

- The compact quantum groups $A_{o}\left(F_{1}\right)$ and $A_{o}\left(F_{2}\right)$ are monoidally equivalent iff $F_{1} \bar{F}_{1}$ and $F_{2} \bar{F}_{2}$ have the same sign and $\operatorname{Tr}\left(F_{1}^{*} F_{1}\right)=\operatorname{Tr}\left(F_{2}^{*} F_{2}\right)$.

- Assume that $A_{o}\left(F_{1}\right)$ and $A_{o}\left(F_{2}\right)$ are monoidally equivalent. Denote by $A_{o}\left(F_{1}, F_{2}\right)$ the universal unital $C^{*}$-algebra generated by the coefficients of

$$
Y \in M_{n_{2}, n_{1}}(\mathbb{C}) \otimes A_{o}\left(F_{1}, F_{2}\right) \quad \text { with relations } \quad Y \quad \text { unitary } \quad \text { and } \quad Y=\left(F_{2} \otimes 1\right) \bar{Y}\left(F_{1}^{-1} \otimes 1\right) .
$$

Then, $A_{o}\left(F_{1}, F_{2}\right) \neq 0$ and there exists a unique pair of commuting universal ergodic actions, $\delta_{1}$ of $A_{o}\left(F_{1}\right)$ and $\delta_{2}$ of $A_{o}\left(F_{2}\right)$, such that

$$
\left(\mathrm{id} \otimes \delta_{1}\right)(Y)=Y_{12}\left(U_{1}\right)_{13} \quad \text { and } \quad\left(\mathrm{id} \otimes \delta_{2}\right)(Y)=\left(U_{2}\right)_{12} Y_{13} .
$$

Here, $U_{i}$ denotes the fundamental representation of $A_{o}\left(F_{i}\right)$.

- $\left(A_{o}\left(F_{1}, F_{2}\right), \delta_{1}, \delta_{2}\right)$ is isomorphic with the $C^{*}$-algebra $B_{u}$ and the actions thereon given by Theorem 4.2

\section{Poisson boundary of the dual of $A_{o}(F)$}

Fix $n \geq 2$ and a matrix $F \in \mathrm{GL}(n, \mathbb{C})$ satisfying $F \bar{F}= \pm 1$. Set $\mathbb{G}=A_{o}(F)$ which remains fixed throughout this section. We assume that $\mathbb{G} \neq \mathrm{SU}_{ \pm 1}(2)$. We identify the Poisson boundary $H^{\infty}(\widehat{\mathbb{G}}, \mu)$ for a generating measure $\mu$ on $\operatorname{Irred}(\mathbb{G})$.

Take the unique $q \in]-1,1\left[\operatorname{such}\right.$ that $F \bar{F}=-\operatorname{sgn} q$ and $\operatorname{Tr}\left(F^{*} F\right)=|q+1 / q|$. By Theorem 4.5, $\mathbb{G}$ is monoidally equivalent with $\mathrm{SU}_{q}(2)=A_{o}\left(F_{q}\right)$, where $F_{q}$ is given by (1.4).

Definition 5.1. We define the $\mathrm{C}^{*}$-algebra $B:=A_{o}\left(F, F_{q}\right)$ and denote by $\beta_{\mathbb{G}}: B \rightarrow B \otimes C(\mathbb{G})$ the ergodic action of $\mathbb{G}$ with invariant state $\omega$, given by Theorem 4.5. So, $B$ is generated by the entries of a unitary 2 by $n$ matrix $Y$ satisfying $Y=F_{q} \bar{Y} F^{-1}$. Define the action $\rho$ of $S^{1}$ on $B$ such that $\rho_{z}(Y)=\left(\begin{array}{c}z \\ 0\end{array}\right) Y$ and set

$$
S^{1} \backslash B:=\left\{x \in B \mid \rho_{z}(x)=x \text { for all } z \in S^{1}\right\} .
$$

The von Neumann algebra generated by $B$ in the GNS-construction for $\omega$ is denoted by $\mathfrak{B}:=(B, \omega)^{\prime \prime}$. By Remark 1.21 (and the co-amenability of $\mathrm{SU}_{q}(2)$ ), the state $\omega$ is a faithful KMS state on $B$ and we regard $B$ as a dense subalgebra of $\mathfrak{B}$.

The generalized Izumi operator is defined as

$$
\Phi: \mathfrak{B} \rightarrow \ell^{\infty}(\widehat{\mathbb{G}}): \Phi(a)=(\operatorname{id} \otimes \omega)\left(\mathbb{X}^{*}(1 \otimes a) \mathbb{X}\right),
$$

where $\mathbb{X}$ is given by $(4.1)$. 
Note that, by Theorem 4.5, the quantum group $\mathrm{SU}_{q}(2)$ admits a (left) ergodic action on $B$. The action $\left(\rho_{z}\right)_{z \in S^{1}}$ is nothing else than the restriction of that ergodic action to the closed subgroup $S^{1}$ of $\mathrm{SU}_{q}(2)$. In this way, $S^{1} \backslash B$ is a higher dimensional counterpart of the Podleś sphere.

Apart from the ergodic action $\beta_{\mathbb{G}}$ of $\mathbb{G}$ on $B$, we also have the analogue of the adjoint action, defined as

$$
\beta_{\widehat{\mathbb{G}}}: \mathfrak{B} \rightarrow \ell^{\infty}(\widehat{\mathbb{G}}) \otimes \mathfrak{B}: \beta_{\widehat{\mathbb{G}}}(x)=\mathbb{X}^{*}(1 \otimes x) \mathbb{X},
$$

where again, $\mathbb{X}$ is given by (4.1). It can be checked easily that $\beta_{\mathbb{G}}$ and $\beta_{\widehat{\mathbb{G}}}$ leave globally invariant $S^{1} \backslash \mathfrak{B}$, yielding actions on $S^{1} \backslash \mathfrak{B}$ that we still denote by $\beta_{\mathbb{G}}, \beta_{\widehat{\mathbb{G}}}$.

The following is the main result of the paper. Its proof takes the rest of the section.

Theorem 5.2. Let $\mathbb{G}=A_{o}(F)$ and let $q$ be as above. Let $\mu$ be a generating measure on $\operatorname{Irred}(\mathbb{G})$. The restriction of the generalized Izumi operator given by (5.1) yields a ${ }^{*}$-isomorphism $\Phi: S^{1} \backslash \mathfrak{B} \rightarrow H^{\infty}(\widehat{\mathbb{G}}, \mu)$. This ${ }^{*}$-isomorphism intertwines the actions $\beta_{\mathbb{G}}, \beta_{\widehat{\mathbb{G}}}$ (see Def. 5.1 and formula (5.2)) with the actions $\alpha_{\mathbb{G}}, \alpha_{\widehat{\mathbb{G}}}$ defined in 2.5 .

Remark 5.3. In order to get a better understanding of the $\mathrm{C}^{*}$-algebra $B$ and the different actions on it, one should look at the case $F=F_{q}$. Then, $B=C\left(\mathrm{SU}_{q}(2)\right)$ and $S^{1} \backslash B$ is exactly the Podleś sphere. Also, the generalized Izumi operator (5.1) coincides with the Izumi operator of Proposition 2.7. Nevertheless, our proof of Theorem 5.2 does not provide an alternative way of identifying the Poisson boundary for the dual of $\mathrm{SU}_{q}(2)$, because Izumi's theorem 2.8 is an ingredient of our proof.

The proof of Theorem 5.2 is given at the end of the section, as a combination of several preliminary results.

- We take a closer look at the generalized Izumi operator $\Phi$ given by (5.1) and prove the equivariance of $\Phi$ with respect to the actions $\beta_{\mathbb{G}}, \beta_{\widehat{\mathbb{G}}}$ and $\alpha_{\mathbb{G}}, \alpha_{\widehat{\mathbb{G}}}$.

- By definition, the generalized Izumi operator $\Phi$ is a normal unital completely positive mapping and we prove that $\Phi$ is multiplicative on $S^{1} \backslash \mathfrak{B}$. The proof uses a technique of [11], which allows to reduce to the case of $\mathbb{G}=\mathrm{SU}_{q}(2)$, where we can apply Theorem 2.8. As it is the case for the other computations of Poisson boundaries of quantum groups in the literature, this is the most subtle part of the proof.

- Once the multiplicativity of $\Phi$ on $S^{1} \backslash \mathfrak{B}$ is proved, the general results gathered in Proposition 2.6 allow to conclude.

\section{Notations and equivariance formulae}

Notation 5.4. All objects related to $\widetilde{\mathbb{G}}=\mathrm{SU}_{q}(2)$ are denoted with tildes, while the corresponding objects related to $\mathbb{G}=A_{o}(F)$ are denoted without tildes. Fix a monoidal equivalence $\varphi: \mathbb{G} \rightarrow \widetilde{\mathbb{G}}$. We identify $\operatorname{Irred}(\mathbb{G})=\operatorname{Irred}(\widetilde{\mathbb{G}})=\mathbb{N}$ and we make once and for all a choice of isometric intertwiners $\widetilde{V}(x \otimes y, z) \in$ $\operatorname{Mor}(x \otimes y, z)$ for $\widetilde{\mathbb{G}}=\mathrm{SU}_{q}(2)$. We take $V(x \otimes y, z)$ such that $\varphi(V(x \otimes y, z))=\widetilde{V}(x \otimes y, z)$.

Whenever $z \in x \otimes y$, we set $p_{z}^{x \otimes y}=V(x \otimes y, z) V(x \otimes y, z)^{*}$ and we define $\widetilde{p}_{z}^{x \otimes y}$ analogously. For any $x \in \mathbb{N}$, we have irreducible representations $U^{x}$ of $\underset{\mathbb{G}}{\mathbb{H}}$ on $H_{x}$ and $\widetilde{U}^{x}$ of $\widetilde{\mathbb{G}}$ on $\widetilde{H}_{x}$. Finally, recall from 1.14, the special states $\psi_{x}$ on $\mathrm{B}\left(H_{x}\right)$ (and hence, $\widetilde{\psi}_{x}$ on $\mathrm{B}\left(\widetilde{H}_{x}\right)$ ).

Proposition 5.5. Let $\Phi$ be as in (5.1) and let $\mu$ be a probability measure on $\operatorname{Irred}(\mathbb{G})$. Then, $\Phi(a) \in$ $H^{\infty}(\widehat{\mathbb{G}}, \mu)$ for all $a \in \mathfrak{B}$.

Proof. Let $a \in \mathfrak{B}$. Then, for $x, y \in \operatorname{Irred}(\mathbb{G})$, we know that

$$
\left(p_{x} \otimes p_{y}\right) \hat{\Delta}(\Phi(a))=\sum_{z \in x \otimes y} V(x \otimes y, z)\left(\Phi(a) p_{z}\right) V(x \otimes y, z)^{*}
$$


So

$$
\begin{aligned}
\left(p_{x} \otimes p_{y}\right) \hat{\Delta}(\Phi(a)) & =\sum_{z \in x \otimes y}(\mathrm{id} \otimes \mathrm{id} \otimes \omega)\left((V(x \otimes y, z) \otimes 1)\left(X^{z}\right)^{*}(1 \otimes a) X^{z}\left(V(x \otimes y, z)^{*} \otimes 1\right)\right) \\
& =\sum_{z \in x \otimes y}(\mathrm{id} \otimes \mathrm{id} \otimes \omega)\left(\left(X_{23}^{y}\right)^{*}\left(X_{13}^{x}\right)^{*}\left(\widetilde{p}_{z}^{x \otimes y} \otimes a\right) X_{13}^{x} X_{23}^{y}\right) \\
& =(\mathrm{id} \otimes \mathrm{id} \otimes \omega)\left(\left(X_{23}^{y}\right)^{*}\left(X_{13}^{x}\right)^{*}(1 \otimes 1 \otimes a) X_{13}^{x} X_{23}^{y}\right)
\end{aligned}
$$

where $(5.3)$ is valid because $(V(x \otimes y, z) \otimes 1)\left(X^{z}\right)^{*}=\left(X^{y}\right)_{23}^{*}\left(X^{x}\right)_{13}^{*}(\widetilde{V}(x \otimes y, z) \otimes 1)$. Then, we get that

$$
\begin{aligned}
p_{x}\left(\mathrm{id} \otimes \psi_{y}\right) \hat{\Delta}(\Phi(a)) & =\left(\mathrm{id} \otimes \psi_{y} \otimes \omega\right)\left(\left(X_{23}^{y}\right)^{*}\left(X_{13}^{x}\right)^{*}(1 \otimes 1 \otimes a) X_{13}^{x} X_{23}^{y}\right) \\
& =(\mathrm{id} \otimes \omega)\left(\left(X^{x}\right)^{*}(1 \otimes a) X^{x}\right)=\Phi(a) p_{x}
\end{aligned}
$$

The equality follows from the fact that $\left(\psi_{y} \otimes \omega\right)\left(\left(X^{y}\right)^{*}(1 \otimes b) X^{y}\right)=\omega(b)$ for $b \in \widetilde{B}$. Indeed, the KMS-property of the state $\omega$ (see (4.2)) gives

$$
\left(\psi_{y} \otimes \omega\right)\left(\left(X^{y}\right)^{*}(1 \otimes b) X^{y}\right)=\left(\widetilde{\psi}_{y} \otimes \omega\right)\left(\left(\widetilde{Q}_{y}^{-2} \otimes 1\right) X^{y}\left(X^{y}\right)^{*}(1 \otimes b)\right)=\left(\widetilde{\varphi}_{y} \otimes \omega\right)(1 \otimes b)=\omega(b)
$$

This completes the proof.

Proposition 5.6. The generalized Izumi operator $\Phi$ defined by (5.1) intertwines the actions $\beta_{\mathbb{G}}, \beta_{\widehat{\mathbb{G}}}$ (see Def. 5.1 and formula (5.2)) with the actions $\alpha_{\mathbb{G}}, \alpha_{\widehat{\mathbb{G}}}$ defined in 2.5 .

Proof. Intertwining of $\alpha_{\mathbb{G}}$ and $\beta_{\mathbb{G}}$ follows from

$$
\begin{aligned}
(\Phi \otimes \mathrm{id}) \beta_{\mathbb{G}}(a) & =(\mathrm{id} \otimes \omega \otimes \mathrm{id})\left(\mathbb{X}_{12}^{*}\left(1 \otimes \beta_{\mathbb{G}}(a)\right) \mathbb{X}_{12}\right)=(\mathrm{id} \otimes \omega \otimes \mathrm{id})\left(\mathbb{V}_{13}\left(\mathrm{id} \otimes \beta_{\mathbb{G}}\right)\left(\mathbb{X}^{*}(1 \otimes a) \mathbb{X}\right) \mathbb{V}_{13}^{*}\right) \\
& =\mathbb{V}\left((\mathrm{id} \otimes \omega \otimes \mathrm{id})\left(\mathrm{id} \otimes \beta_{\mathbb{G}}\right)\left(\mathbb{X}^{*}(1 \otimes a) \mathbb{X}\right)\right) \mathbb{V}^{*}=\mathbb{V}\left((\mathrm{id} \otimes \omega)\left(\mathbb{X}^{*}(1 \otimes a) \mathbb{X}\right) \otimes 1\right) \mathbb{V}^{*} \\
& =\alpha_{\mathbb{G}}(\Phi(a)) .
\end{aligned}
$$

On the other hand, intertwining of $\alpha_{\widehat{\mathbb{G}}}$ and $\beta_{\widehat{\mathbb{G}}}$ is a consequence of

$$
\begin{aligned}
\alpha_{\widehat{\mathbb{G}}}(a) V(y \otimes z, x) & =\widehat{\Delta}(\Phi(a)) V(y \otimes z, x)=V(y \otimes z, x) \Phi(a) p_{x}=V(y \otimes z, x)(\mathrm{id} \otimes \omega)\left(\left(X^{x}\right)^{*}(1 \otimes a) X^{x}\right) \\
& =(\mathrm{id} \otimes \mathrm{id} \otimes \omega)\left(\left(X^{z}\right)_{23}^{*}\left(X^{y}\right)_{13}^{*}(1 \otimes 1 \otimes a) X_{13}^{y} X_{23}^{z}\right) V(y \otimes z, x) \\
& =(\mathrm{id} \otimes \Phi)\left(\mathbb{X}^{*}(1 \otimes a) \mathbb{X}\right) V(y \otimes z, x)=(\mathrm{id} \otimes \Phi) \beta_{\widehat{\mathbb{G}}}(a) V(y \otimes z, x)
\end{aligned}
$$

for every $x, y, z \in \operatorname{Irred}(\mathbb{G})$.

\section{Multiplicativity of the generalized Izumi operator}

Fix a generating probability measure $\mu$ on $\operatorname{Irred}(\mathbb{G})$. We prove that the generalized Izumi operator $\Phi$ given by (5.1), is multiplicative on $S^{1} \backslash \mathfrak{B}$, using a strategy from [11].

For every $y \in \operatorname{Irred}(\mathbb{G})$, we define

$$
\Phi_{y}: \mathfrak{B} \rightarrow B\left(H_{y}\right): \Phi_{y}(a):=\Phi(a) p_{y}=(\mathrm{id} \otimes \omega)\left(\left(X^{y}\right)^{*}(1 \otimes a) X^{y}\right)
$$

Lemma 5.7. For the scalar products on $\mathfrak{B}$ and $B\left(H_{y}\right)$ given respectively by $\omega$ and $\psi_{y}$, the linear map $\Phi_{y}$ has an adjoint mapping $\Phi_{y}^{*}: B\left(H_{y}\right) \rightarrow \mathfrak{B}$ given by

$$
\Phi_{y}^{*}(b)=\left(\widetilde{\varphi}_{y} \otimes \mathrm{id}\right)\left(X^{y}(b \otimes 1)\left(X^{y}\right)^{*}\right)
$$

So $\omega\left(a \Phi_{y}^{*}(b)\right)=\psi_{y}\left(\Phi_{y}(a) b\right)$ for $a \in \mathfrak{B}$ and $b \in B\left(H_{y}\right)$. 
Proof. Again, we use the KMS-property of $\omega$. From this it follows that

$$
\begin{aligned}
\psi_{y}\left(\Phi_{y}(a) b\right) & \left.=\psi_{y}(\mathrm{id} \otimes \omega)\left(\left(X^{y}\right)^{*}(1 \otimes a) X^{y} b\right)\right)=\left(\psi_{y} \otimes \omega\right)\left(\left(X^{y}\right)^{*}(1 \otimes a) X^{y}(b \otimes 1)\right) \\
& =\left(\widetilde{\varphi}_{y} \otimes \omega\right)\left((1 \otimes a) X^{y}(b \otimes 1)\left(X^{y}\right)^{*}\right)=\omega\left(a\left(\widetilde{\varphi}_{y} \otimes \mathrm{id}\right)\left(X^{y}(b \otimes 1)\left(X^{y}\right)^{*}\right)\right) \\
& =\omega\left(a \Phi_{y}^{*}(b)\right)
\end{aligned}
$$

Remark 5.8. We already know that $\left(\Phi_{y} \otimes \mathrm{id}\right) \beta_{\mathbb{G}}=\alpha_{\mathbb{G}} \circ \Phi_{y}$. From the definition of the adjoint $\Phi_{y}^{*}$ it follows that $\left(\Phi_{y}^{*} \otimes \mathrm{id}\right) \alpha_{\mathbb{G}}=\beta_{\mathbb{G}} \circ \Phi_{y}^{*}$. This follows also from the next calculation.

$$
\begin{aligned}
\left(\Phi_{y}^{*} \otimes \mathrm{id}\right) \alpha_{\mathbb{G}}(a) & =\left(\widetilde{\varphi}_{y} \otimes \mathrm{id} \otimes \mathrm{id}\right)\left(X_{12}^{y}\left(\alpha_{\mathbb{G}}(a)\right)_{13}\left(X_{12}^{y}\right)^{*}\right)=\left(\widetilde{\varphi}_{y} \otimes \mathrm{id} \otimes \mathrm{id}\right)\left(X_{12}^{y} V_{13}(a \otimes 1 \otimes 1) V_{13}^{*}\left(X_{12}^{y}\right)^{*}\right) \\
& =\left(\widetilde{\varphi}_{y} \otimes \mathrm{id} \otimes \mathrm{id}\right)\left(\left(\mathrm{id} \otimes \beta_{\mathbb{G}}\right)\left(X^{y}(a \otimes 1)\left(X^{y}\right)^{*}\right)\right)=\beta_{\mathbb{G}}\left(\left(\widetilde{\varphi}_{y} \otimes \mathrm{id}\right)\left(X^{y}(a \otimes 1)\left(X^{y}\right)^{*}\right)\right) \\
& =\beta_{\mathbb{G}}\left(\Phi_{y}^{*}(a)\right)
\end{aligned}
$$

Lemma 5.9. Let $\mu$ be a generating probability measure on $\operatorname{Irred}(\mathbb{G})$. Denote

$$
\mathcal{P}_{y}=\Phi_{y}^{*} \Phi_{y}: \mathfrak{B} \rightarrow \mathfrak{B} \quad \text { and } \quad \mathcal{P}_{\mu}=\sum_{y} \mu(y) \mathcal{P}_{y}
$$

The sequence $\left(\mathcal{P}_{\mu^{* n}}\right)_{n}$ converges pointwize ${ }^{*}$-strongly to a completely positive unital map $\mathcal{P}_{\infty}: \mathfrak{B} \rightarrow \mathfrak{B}$. Moreover, if $a \in \mathfrak{B}$, the following are equivalent.

- The element a belongs to the multiplicative domain of the completely positive unital map $\Phi$.

- We have $\mathcal{P}_{\infty}(a)=a$.

Proof. We first make the following claim.

Claim. For every $a \in \mathfrak{B}$, the sequence $\mathcal{P}_{\mu^{* n}}(a)$ converges ${ }^{*}$-strongly. Denoting its limit as $\mathcal{P}_{\infty}(a)$, we have

$$
\widehat{\varepsilon}(\Phi(b) \cdot \Phi(a))=\omega\left(b \mathcal{P}_{\infty}(a)\right) \text { for all } a, b \in \mathfrak{B} .
$$

We prove the claim below and argue already how the lemma follows from it. If $a \in \mathfrak{B}$ belongs to the multiplicative domain of $\Phi$, we have $\Phi(b) \cdot \Phi(a)=\Phi(b a)$ for all $b \in \mathfrak{B}$. The formula in the claim yields $\omega(b a)=\widehat{\varepsilon}(\Phi(b a))=\omega\left(b \mathcal{P}_{\infty}(a)\right)$ for all $b \in \mathfrak{B}$. Hence, $\mathcal{P}_{\infty}(a)=a$. Conversely, if $\mathcal{P}_{\infty}(a)=a$, we find that $\widehat{\varepsilon}\left(\Phi(a)^{*} \cdot \Phi(a)\right)=\widehat{\varepsilon}\left(\Phi\left(a^{*} a\right)\right)$. Since $\Phi(a)^{*} \cdot \Phi(a) \leq \Phi\left(a^{*} a\right)$ and since $\widehat{\varepsilon}$ is faithful on $H^{\infty}(\widehat{\mathbb{G}}, \mu)$, it follows that $\Phi(a)^{*} \cdot \Phi(a)=\Phi\left(a^{*} a\right)$. This implies that $a$ belongs to the multiplicative domain of $\Phi$.

It remains to prove the claim. For all $a, b \in \mathfrak{B}$, we have

$$
\begin{aligned}
\widehat{\varepsilon}(\Phi(b) \cdot \Phi(a)) & =\widehat{\varepsilon}\left(\lim _{n \rightarrow \infty} P_{\mu^{* n}}(\Phi(b) \Phi(a))\right)=\lim _{n \rightarrow \infty} \psi_{\mu^{* n}}(\Phi(b) \Phi(a)) \\
& =\lim _{n \rightarrow \infty} \sum_{x} \mu^{* n}(x) \psi_{x}\left(\Phi_{x}(b) \Phi_{x}(a)\right)=\lim _{n \rightarrow \infty} \omega\left(b \mathcal{P}_{\mu^{* n}}(a)\right) .
\end{aligned}
$$

It follows that $\mathcal{P}_{\mu^{* n}}(a)$ is weakly convergent, say to $\mathcal{P}_{\infty}(a) \in \mathfrak{B}$. Since all the $\mathcal{P}_{\mu^{* n}}$ and $\mathcal{P}_{\infty}$ commute with the ergodic action $\beta_{\mathbb{G}}$ of $\mathbb{G}$ on $\mathfrak{B}$ and preserve the state $\omega$, these completely positive operators preserve the (finite-dimensional) spectral subspaces of $\beta_{\mathbb{G}}$ and it follows that $\mathcal{P}_{\mu^{* n}}(a) \rightarrow \mathcal{P}_{\infty}(a)^{*}$-strongly. This proves the claim and ends the proof of the lemma.

With respect to the ergodic action $\beta_{\mathbb{G}}$, the von Neumann algebra $\mathfrak{B}$ has a natural dense ${ }^{*}$-subalgebra given as the linear span of the spectral subspaces $B_{x}, x \in \operatorname{Irred}(\mathbb{G})$. Since $\mathcal{P}_{y}$ commutes with $\beta_{\mathbb{G}}$, it follows that $\mathcal{P}_{y}$ maps $B_{x}$ into $B_{x}$. We study more closely this operator on the finite-dimensional vector space $B_{x}$.

Denote as follows the restriction of the operator $\Phi_{y}$ to the spectral subspace $B_{x}$

$$
\Phi_{y}^{x}: B_{x} \rightarrow B\left(H_{y}\right): \Phi_{y}^{x}(a)=\Phi(a) p_{y} .
$$


We use the following unitary identifications that are consequences of Notation 1.14 and formula (4.3).

$$
\begin{aligned}
& v_{y}:\left(B\left(H_{y}\right), \psi_{y}\right) \rightarrow H_{y} \otimes H_{y}: A \mapsto \frac{1}{\sqrt{\operatorname{dim}_{q}(y)}}(A \otimes 1) t_{y} \\
& \phi_{x}:\left(B_{x}, \omega\right) \rightarrow H_{x} \otimes \widetilde{\widetilde{H}}_{x}:\left(\omega_{\tilde{\mu}, \rho} \otimes \mathrm{id}\right)\left(X^{x}\right) \mapsto \frac{1}{\sqrt{\operatorname{dim}_{q}(x)}} \rho \otimes \overline{\widetilde{Q}_{x}^{-1 / 2} \widetilde{\mu}}
\end{aligned}
$$

with $\rho \in H_{x}$ and $\widetilde{\mu} \in \widetilde{H}_{x}$.

Lemma 5.10. For every $x, y \in \operatorname{Irred}(\mathbb{G})$, we have

$$
v_{y} \circ \Phi_{y}^{x} \circ \phi_{x}^{*}=V(y \otimes y, x)\left(1 \otimes{\overline{\xi_{y}^{x}}}^{*}\right)
$$

where the vector $\xi_{y}^{x} \in \widetilde{H}_{x}$ is defined as

$$
\xi_{y}^{x}=\frac{1}{\sqrt{\operatorname{dim}_{q}(x) \operatorname{dim}_{q}(y)}} \widetilde{V}(y \otimes y, x)^{*}\left(\widetilde{Q}_{y}^{-2} \otimes 1\right) \tilde{t}_{y} .
$$

Proof. Take $a \in B\left(H_{y}\right)$ and $b \in B_{x}$. By Lemma 5.7, we have

$$
\psi_{y}\left(a^{*} \Phi_{y}^{x}(b)\right)=\omega\left(\left(\widetilde{\varphi}_{y} \otimes \mathrm{id}\right)\left(X^{y}(a \otimes 1)\left(X^{y}\right)^{*}\right)^{*} b\right) .
$$

Further calculation gives

$$
\begin{aligned}
\left(\widetilde{\varphi}_{y} \otimes \mathrm{id}\right)\left(X^{y}(a \otimes 1)\left(X^{y}\right)^{*}\right) & =\left(\widetilde{\psi}_{y} \otimes \mathrm{id}\right)\left(\left(\widetilde{Q}_{y}^{-2} \otimes 1\right) X^{y}(a \otimes 1)\left(X^{y}\right)^{*}\right) \\
& =\frac{1}{\operatorname{dim}_{q}(y)}\left(\tilde{t}_{y}^{*} \otimes 1\right)\left(\widetilde{Q}_{y}^{-2} \otimes 1 \otimes 1\right) X_{13}^{y}(a \otimes 1 \otimes 1)\left(X_{13}^{y}\right)^{*}\left(\tilde{t}_{y} \otimes 1\right) \\
& =\frac{1}{\sqrt{\operatorname{dim}_{q}(y)}}\left(\tilde{t}_{y}^{*} \otimes 1\right)\left(\widetilde{Q}_{y}^{-2} \otimes 1 \otimes 1\right) X_{13}^{y} X_{23}^{y}\left(v_{y}(a) \otimes 1\right) .
\end{aligned}
$$

We know that

$$
X_{13}^{y} X_{23}^{y}=\sum_{z \in y \otimes y}(\widetilde{V}(y \otimes y, z) \otimes 1) X^{z}\left(V(y \otimes y, z)^{*} \otimes 1\right) .
$$

So, $\psi_{y}\left(a^{*} \Phi_{y}^{x}(b)\right)=\omega\left(D^{*} b\right)$ where

$$
D=\frac{1}{\sqrt{\operatorname{dim}_{q}(y)}}\left(\tilde{t}_{y}^{*}\left(\widetilde{Q}_{y}^{-2} \otimes 1\right) \widetilde{V}(y \otimes y, x) \otimes 1\right) X^{x}\left(V(y \otimes y, x)^{*} v_{y}(a) \otimes 1\right) .
$$

Here, only the term $z=x$ in the sum (5.5) remained because of the orthogonality relations (4.3) and the assumption $b \in B_{x}$.

Using the commutation relations $\left(\widetilde{Q}_{y}^{-1 / 2} \otimes \widetilde{Q}_{y}^{-1 / 2}\right) \widetilde{V}(y \otimes y, x)=\widetilde{V}(y \otimes y, x) \widetilde{Q}_{x}^{-1 / 2}$ and $\left(\widetilde{Q}_{y}^{-1 / 2} \otimes \widetilde{Q}_{y}^{-1 / 2}\right) \tilde{t}_{y}=\tilde{t}_{y}$ and the formula $\omega\left(D^{*} b\right)=\left\langle\phi_{x}(D), \phi_{x}(b)\right\rangle$, we arrive at

$$
\psi_{y}\left(a^{*} \Phi_{y}^{x}(b)\right)=\left\langle V(y \otimes y, x)^{*} v_{y}(a) \otimes \overline{\xi_{y}^{x}}, \phi_{x}(b)\right\rangle
$$

where $\xi_{y}^{x}$ is given in the statement of the lemma. This proves the lemma.

We finally prove the multiplicativity of the operator $\Phi$ on $S^{1} \backslash \mathfrak{B}$.

Lemma 5.11. The elements of $S^{1} \backslash \mathfrak{B}$ belong to the multiplicative domain of the generalized Izumi operator $\Phi$ introduced in (5.1). 
Proof. Since the action $\beta_{\mathbb{G}}$ on $\mathfrak{B}$ commutes with the action $\rho$ of $S^{1}$ on $\mathfrak{B}$, it suffices to show that every element of $B_{x}$ that is invariant under $\rho$ belongs to the multiplicative domain of $\Phi$.

Observe that for $z=|q|^{i t}$, we have $\phi_{x} \rho_{z}=\left(1 \otimes \widetilde{Q}^{-i t}\right) \phi_{x}$. Combining Lemmas 5.9 and 5.10 , the multiplicativity of $\Phi$ on $S^{1} \backslash \mathfrak{B}$ is then equivalent with the statement

$$
\lim _{n \rightarrow \infty} \sum_{y} \mu^{* n}(y) \xi_{y}^{x}\left\langle\xi_{y}^{x}, \eta\right\rangle \rightarrow \eta \text { for all } \eta \in \widetilde{H}_{x}^{S^{1}},
$$

where we denote by $\widetilde{H}_{x}^{S^{1}}$ the subspace of vectors $\eta \in \widetilde{H}_{x}$ satisfying $Q_{x} \eta=\eta$.

This last statement concerns only $\mathrm{SU}_{q}(2)$ and hence holds because of Theorem 2.8.

\section{Proof of Theorem 5.2}

We have gathered enough material to give the

Proof of Theorem 5.2. By Proposition 5.5 and Lemma 5.11, we have the normal unital *-homomorphism

$$
\Phi: S^{1} \backslash \mathfrak{B} \rightarrow H^{\infty}(\widehat{\mathbb{G}}, \mu) .
$$

Because $\omega$ is faithful, the map $\Phi$ is injective and by Proposition 5.6, $\Phi$ intertwines the actions $\beta_{\mathbb{G}}$, $\beta_{\widehat{\mathbb{G}}}$ on $S^{1} \backslash \mathfrak{B}$ with the actions $\alpha_{\mathbb{G}}, \alpha_{\widehat{\mathbb{G}}}$ on $H^{\infty}(\widehat{\mathbb{G}}, \mu)$. So, it remains to prove the surjectivity of $\Phi$.

From Proposition 2.6, we know that $\alpha_{\mathbb{G}}$ is an ergodic action of $\mathbb{G}$ on $H^{\infty}(\widehat{\mathbb{G}}, \mu)$ and that $\operatorname{mult}\left(x, \alpha_{\mathbb{G}}\right)=0$ for $x$ odd, $\operatorname{mult}\left(x, \alpha_{\mathbb{G}}\right) \leq 1$ for $x$ even. On the other hand, the multiplicity of $x$ in the restriction of $\beta_{\mathbb{G}}$ to $S^{1} \backslash \mathfrak{B}$ is exactly 1 for $x$ even. Indeed, as we have seen in the proof of Lemma 5.11, $v_{x}$ maps the $S^{1}$-invariant elements of $B_{x}$ onto $H_{x} \otimes \overline{\widetilde{H}_{x}^{S^{1}}}$ and $\widetilde{H}_{x}^{S^{1}}$ is one-dimensional for $x$ even. Indeed, the eigenvalues of $\widetilde{Q}_{x}$ are $q^{x}, q^{x-2}, \ldots, q^{2-x}, q^{-x}$, all of multiplicity 1 on the Hilbert space $\widetilde{H}_{x}$ of dimension $x+1$. Injectivity and equivariance of $\Phi$ finish the proof.

\section{Martin boundary of the dual of $A_{o}(F)$}

We prove an identification theorem for the Martin boundary of the dual of $A_{o}(F)$.

As above, fix $\mathbb{G}=A_{o}(F)$ with $\mathbb{G} \neq \mathrm{SU}_{ \pm 1}(2)$. Take $q$, the $\mathrm{C}^{*}$-algebra $B$ and the actions $\beta_{\mathbb{G}}$ and $\beta_{\widehat{\mathbb{G}}}$ as in Definition 5.1 and formula (5.2). The $\mathrm{C}^{*}$-algebra $B$ admits the natural action $\left(\rho_{z}\right)_{z \in S^{1}}$ of $S^{1}$ and the subalgebra of $S^{1}$-invariant elements was denoted by $S^{1} \backslash B$.

Recall from (3.1) that the Martin boundary $A_{\mu}$ is naturally equipped with actions $\gamma_{\mathbb{G}}$ and $\gamma_{\widehat{\mathbb{G}}}$.

Theorem 6.1. Let $\mu$ be a generating measure on $\operatorname{Irred}(\mathbb{G})$ that is transient and has finite first moment:

$$
\sum_{x \in \mathbb{N}} x \mu(x)<\infty .
$$

The composition of the generalized Izumi operator given in (5.1) and the quotient map $\pi: \ell^{\infty}(\widehat{\mathbb{G}}) \rightarrow \frac{\ell^{\infty}(\widehat{\mathbb{G}})}{c_{0}(\widehat{\mathbb{G}})}$ defines $a{ }^{*}$-isomorphism

$$
\pi \circ \Phi: S^{1} \backslash B \rightarrow A_{\mu}
$$

of $S^{1} \backslash B$ onto the Martin boundary $A_{\mu}$. This ${ }^{*}$-isomorphism intertwines the actions $\beta_{\mathbb{G}}, \beta_{\widehat{\mathbb{G}}}$ on $S^{1} \backslash B$ with the actions $\gamma_{\mathbb{G}}, \gamma_{\widehat{\mathbb{G}}}$ on $A_{\mu}$. 
Proof. Combining Theorems 5.6 and 5.8 in [16], $A_{\mu}$ admits a state $\omega_{\infty}$ such that the map

$$
T: A_{\mu} \rightarrow H^{\infty}(\widehat{\mathbb{G}}, \mu): T(a)=\left(\operatorname{id} \otimes \omega_{\infty}\right) \gamma_{\widehat{\mathbb{G}}}(a)
$$

is a ${ }^{*}$-homomorphism with dense range. Moreover, by formula (5.3) in [16], the composition $\pi \circ T$ is the identity map. Since $T$ intertwines the action $\gamma_{\mathbb{G}}$ on $A_{\mu}$ with the action $\alpha_{\mathbb{G}}$ on $H^{\infty}(\widehat{\mathbb{G}}, \mu)$, it follows that $T$ is a *-isomorphism of $A_{\mu}$ onto the $\mathrm{C}^{*}$-algebra $D$ defined as the closed linear span of the spectral subspaces of the action $\alpha_{\mathbb{G}}$ of $\mathbb{G}$ on $H^{\infty}(\widehat{\mathbb{G}}, \mu)$. Moreover, the quotient map $\pi$ provides the inverse of this *-isomorphism. By Theorem 5.2, the restriction of the generalized Izumi operator, yields the ${ }^{*}$-isomorphism $\Phi: S^{1} \backslash B \rightarrow D$. Composing $\pi$ and $\Phi$, we are done.

\section{References}

[1] T. BAnica, Théorie des représentations du groupe quantique compact libre $O(n)$. C. R. Acad. Sci. Paris Sér. I Math. 322 (1996), 241-244.

[2] T. Banica, Le groupe quantique compact libre U(n). Commun. Math. Phys. 190 (1997), 143-172.

[3] P. Biane, Marches de Bernoulli quantiques. In Séminaire de Probabilités, XXIV, 1988/89, Lecture Notes in Math. 1426, Springer, Berlin, 1990, pp. 329-344.

[4] P. Biane, Quantum random walk on the dual of SU(n). Probab. Th. Rel. Fields 89 (1991), 117-129.

[5] P. Biane, Théorème de Ney-Spitzer sur le dual de SU(2). Trans. Amer. Math. Soc. 345 (1994), $179-194$.

[6] J. Bichon, A. De Rijdt \& S. VAes, Ergodic coactions with large quantum multiplicity and monoidal equivalence of quantum groups. Comm. Math. Phys 262 (2006), 703-728.

[7] B. Collins, Martin boundary theory of some quantum random walks. Ann. Inst. H. Poincaré Probab. Statist. 40 (2004), 367-384.

[8] E.G. Effros \& Z.-J. Ruan, Discrete quantum groups. I. The Haar measure. Internat. J. Math. 5 (1994), 681-723.

[9] T. Hayashi, Harmonic function spaces of probability measures on fusion algebras. Publ. Res. Inst. Math. Sci. 36 (2000), 231-252.

[10] M. Izumi, Non-commutative Poisson boundaries and compact quantum group actions. Adv. Math. 169 (2002), 1-57.

[11] M. Izumi, S. Neshveyev \& L. Tuset, Poisson boundary of the dual of $\mathrm{SU}_{q}(n)$. Comm. Math. Phys. 262 (2006), 505-531.

[12] V.A. Kaimanovich, Boundaries of invariant Markov operators: the identification problem. In Ergodic theory of $\mathbb{Z}^{d}$ actions (Warwick, 1993-1994), London Math. Soc. Lecture Note Ser. 228, Cambridge Univ. Press, Cambridge, 1996, pp. 127-176.

[13] A. Maes \& A. Van Daele, Notes on compact quantum groups. Nieuw Arch. Wisk. (4) 16 (1998), $73-112$.

[14] S. Neshveyev \& L. Tuset, The Martin boundary of a discrete quantum group. J. Reine Angew. Math. 568 (2004), 23-70.

[15] P. Podleś, Symmetries of quantum spaces. Subgroups and quotient spaces of quantum SU(2) and SO(3) groups. Comm. Math. Phys. 170 (1995), 1-20. 
[16] S. Vaes \& R.Vergnioux, The boundary of universal discrete quantum groups, exactness and factoriality. Duke Math. J., to appear. math.0A/0509706

[17] A. Van Daele, Discrete quantum groups. J. Algebra 180 (1996), 431-444.

[18] A. Van Daele \& S. Wang, Universal quantum groups. Internat. J. Math. 7 (1996), 255-263.

[19] S.L. Woronowicz, Compact quantum groups. In Symétries quantiques (Les Houches, 1995), NorthHolland, Amsterdam, 1998, pp. 845-884.

[20] S.L. Woronowicz, Twisted SU(2) group. An example of a non-commutative differential calculus. Publ. Res. Inst. Math. Sci. 23 (1987), 117-181. 